COMMENTARY

\section{Inflammatory Breast Cancer Disease: A Literature Review}

\author{
Patricia Y. Talbert* and Marquise D. Frazier
}

Howard University, USA

\begin{abstract}
Physicians, health care practitioners, and breast cancer advocacy groups have spent many decades investing in the promotion of breast cancer screening and early detection mechanisms, such as mammograms and breast self-examinations. These screening modalities are extremely important in reducing breast cancer incidence, prevalence, and mortality, as breast cancer screening continues to contribute to decreasing the surging mortality rate of cancerous diseases. Without the use of these screening measures, breast cancer mortality would affect many more women and exceed current statistics. While these screenings are important for the early detection of breast cancer, a more uncommon, virulent, and fatal form of breast cancer known as Inflammatory Breast Cancer (IBC) has gone largely unreported and continues to affect women. Considering IBC is an aggressive form of breast cancer with a five-year survival rate of 65\%, more diligent measures and strategies are needed to educate women throughout the world about IBC. The purpose of this review is to present an overview of the literature introducing IBC, address symptoms to spread awareness, concentrate on early detection, and focus on the comprehensive treatment options of IBC.
\end{abstract}

\section{Keywords}

Inflammatory Breast Cancer, chemotherapy, radiation, double mastectomy, modality management

\section{Introduction}

Health care practitioners have spent many decades promoting preventive screening, such Open Access

Citation: Talbert, PY, Frazier MD. Inflammatory Breast Cancer Disease: A Literature Review. Cancer Studies. 201; 2(1):4.

Received: August 07, 2019 Accepted: October 24, 2019 Published: November 25, 2019

Copyright: () 2019 Talbert PY. This is an open access article distributed under the terms of the Creative Commons Attribution License, which permits unrestricted use, distribution, and reproduction in any medium, provided the original author and source are credited.

\section{Corresponding author:}

Patricia Y. Talbert, Howard University, USA.

E-mail: patricia.talbert@howard.edu as mammography testing and self-breast examinations (SBE). For certain, mammograms, clinical examinations, and SBE are of extremely important in reducing breast cancer and decreasing the surging mortality of this disease. Without the use of these preventive measures, breast cancer mortality would affect many more women and succeed the estimated 268,000 new cases of breast cancer in 2019 and the 42,260 that succumb (i.e., 41,760 women, 500 men) to this disease. ${ }^{1}$ The purpose of this review is to present an overview of the literature introducing Inflammatory Breast Cancer (IBC), address symptoms to spread awareness, concentrate on early detection, and focus on the comprehensive treatment options of IBC.

There is an uncommon type of breast cancer, called Inflammatory Breast Cancer (IBC) that is rare and a very aggressive form of breast cancer accounting for " $2-4 \%$ of all breast cancer cases (despite its low incidence) and "contributes to $7 \%$ to $10 \%$ of breast cancer caused mortality". ${ }^{2}$ As noted by the National Cancer Institute this disease occurs when cancer cells block the lymph vessels in the skin of the breast, causing the breast to become red, swollen, and warm. ${ }^{3}$ Further, Mei and Merajver included other salient characteristics of IBC, such as "rapid progression and poor survival compared to non-IBC breast cancer" (p. 25). ${ }^{4}$ It is also a disease that is understudied, has poor outcomes (because it can be misdiagnosed resulting in delayed treatment), which are all precursors to a potential poor prognosis. Considering the carcinogenesis of IBC, it can be extremely challenging for healthcare practitioners and patients to recognize this disease immediately. Consequently, further dialogue is needed to 
educate and inform individuals about IBC, funding is also needed to support additional research to support prevention, and create new regimens to improve outcomes of IBC.

All of these recommendations would contribute to an increase in clinicians' ability to support patients and continue to have educational dialogue to combat this uncommon and rare form of breast cancer, unlike non-IBCs such as triple negative breast cancer (TNBC).

\section{Detection of Inflammatory Breast Cancer}

The disease is unique in that IBC does not produce a distinct mass or lump that can be felt by BSE or detected by a mammogram, which is what most women have been taught over the years. Since this disease does not present as common forms of breast cancer often do, such as palpated lumps on BSE, it can be extremely difficult to diagnose, and as a result, it is even more important to educate and empower women with knowledge regarding IBC. In regard to detection, a mammogram can detect some skin thickening, tumors, and swelling or abnormal enlargement of the lymph nodes within many women. Thus, this is reassuring in a sense because the preventative technique has some capacity in possibly finding abnormalities. Unfortunately, because IBC spreads rapidly to other parts of the body, early detection and treatment is vital in order to increase the probability of surviving this disease. In a sense, although mammograms are essential for detection, the best means of exposing this disease is through knowledge regarding the cause of IBC and specific characteristics of this disease.

\section{Method}

The reviewed studies were identified through PubMed, CINAHL, Google Scholar, and Science Direct research using the combination of the following terms for English, French, and Spanish languages: inflammatory breast cancer, breast cancer, neoadjuvant chemotherapy, radiation therapy, double mastectomy, modality management, clinical presentation, molecular profile, and treatment. For each study, abstracts were read and included based on there relevance and they had to meet the following criteria: 1) To be focused on IBC; 2) To provide comprehensive information of the diagnosis; 3 ) To consider treatment plan and care; and 4) To be majorly based on the recent studies and within the last 15 years. Accordingly, selected studies were then read and evaluated for inclusion. Only studies on breast cancer survivorship, behavior, and quality of life were included. Given that this pathology is rare, references of the selected studies were also reviewed and considered for inclusion. At the end, only 20 articles and abstracts were included in the examination of this literature review and 13 articles providing similar information but contained older information.

\section{Risk Factors and Symptoms of IBC}

While generally non-IBC is presented through cancer screening or by the presence of a breast-lump and/or pain, the clinical presentation of IBC can be introduced in many different forms, which can be confusing, frequently overlooked, and misdiagnosed in the early stage and/or in general. There are many epidemiological studies that provided additional insight regarding the risk factors and symptoms of IBC. For instance, Menta and colleagues introduced that high body mass index continues to be recognized as an independent risk factor of IBC. Whereas, the National Cancer Institute explained, unlike the more common types of breast cancer, IBC generally does not display or show-up as a lump, partially because the disease grows as nests under the skin, and therefore, it can hide and lay dormant for long periods, bacteria can mature, disperse, and grant an opportunity for it to spread causing neighboring cells to form further IBCs, plus, once surface, it can be a rapid onset of IBC. . $3,5,6^{-1}$ This is a major reason why IBC can be frequently overlooked by so many. Highly critical and similar to findings from many other scholars, Taghian and Merajver underscored that IBC usually does not produce a lump that can be felt within the breast; instead it causes thickening, swelling, reddened and warm to the touch, or may resemble the texture of an orange peel (peau d'orange), and painful and enlarged breasts, and appears inflamed. ${ }^{7}$ Likewise, (Table 1.) presented by the Susan G. Komen Breast Cancer Institute $(2013 ; 2019)$ lists several relevant symptoms of inflammatory breast cancer. ${ }^{8,9}$ Considering that IBC is rare with multiple possible symptoms, detailed conversations are vital to change the status quo. These symptoms may vary, and one may occur without the other. However, if any of these symptoms should occur, it is extremely important for women to see their physician and be aggressive regarding their care choices. This approach can be a life-saving measure that can make a difference. IBC is a type of cancer that can move quickly and change rapidly unlike non-IBCs. 
Table 1. Symptoms of Inflammatory Breast Cancer

\begin{tabular}{|c|c|}
\hline IBC Symptoms & Notes \\
\hline One breast becomes much larger than the other one & (often sudden) \\
\hline Warmth and swelling in the breast & (often sudden) \\
\hline \multicolumn{2}{|l|}{ Redness or pinkness that may look like an infection } \\
\hline \multicolumn{2}{|l|}{ Itching or pain in the breast that won't go away } \\
\hline \multicolumn{2}{|l|}{ Dimpling of the skin that may look like the skin of } \\
\hline \multicolumn{2}{|l|}{ An orange (called peaud'orange) } \\
\hline \multicolumn{2}{|l|}{ Ridges or thickened areas of skin } \\
\hline \multicolumn{2}{|l|}{ Nipple discharge /Nipple retraction or flattening } \\
\hline Change in the color of the areola & (the dark skin around the nipple) \\
\hline \multicolumn{2}{|l|}{ A bruise that does not go away } \\
\hline Swollen lymph nodes on the neck or under the arm & (although often there is no lump) \\
\hline \multicolumn{2}{|l|}{ A lump } \\
\hline \multicolumn{2}{|c|}{ Note: The Susan G. Komen Breast Cancer Institute (2013; 2019). Inflammatory Breast Cancer. } \\
\hline \multicolumn{2}{|l|}{ Treatment Options } \\
\hline \multicolumn{2}{|c|}{$\begin{array}{l}\text { Early detection is most desirable and increases the chance of surviving IBC and undergoing } \\
\text { an effective treatment. An individual's treatment is based on multiple factors, such as the stage } \\
\text { of tumor (which is most crucial), age, and the many etiological risk factors associated. These } \\
\text { factors are all imperative for increasing the patient's prognosis of surviving IBC. As technology } \\
\text { continues to improve and medical advancements are made in the realm of oncology, } \\
\text { researchers have concluded the best mechanisms for treatment of IBC begins with } \\
\text { chemotherapy, and is typically followed by surgery and/or radiation with the determination on } \\
\text { next steps being tumor response to chemotherapeutic agents. Surgery and radiation may be } \\
\text { used to target the remaining cancerous cells within the breast and attack possible cancer that } \\
\text { has spread under the arm to the adjacent nodal region. }{ }^{1,3} \text { The treatment combination depends } \\
\text { on what is best for the patient and the most effective modalities to achieve the best outcome to } \\
\text { minimize any potential metastasis and eradicate the disease. A standard protocol for } \\
\text { treatment of IBC might include a combination of strategies, to include chemotherapy, surgery, } \\
\text { and radiation therapy. The overall benefit is this combined modality approach, which can allow } \\
\text { for better control of IBC compared to many decades ago. The most common approach to } \\
\text { treatment IBC can start with a chemotherapy protocol and regimen. }\end{array}$} \\
\hline
\end{tabular}

\section{Chemotherapy}

Systemic and targeted chemotherapies are usually administered in a neoadjuvant fashion that is prior to surgery or radiation therapy. Medical oncologists utilize many different classes of chemotherapeutic agents to include anthracyclines, taxanes (targeted chemotherapeutic agents), alkalating agents, and pyrimidine analogs. ${ }^{10}$ According to van Uden and colleagues, three cycles of systemic neoadjuvant chemotherapy were administered to 68 patients, followed by surgery and six additional cycles of adjuvant chemotherapy and radiation therapy treatments, which yielded significant long-term survival rates of approximately $32-44 \%$, with this combined modality approach. ${ }^{10}$ Another point of view has been discussed by Beaver, Williamson, and Briggs by exploring the experience of women who received neo-adjuvant chemotherapy for breast cancer. ${ }^{11}$ The authors interviewed 20 women who had completed neo-adjuvant chemotherapy for breast cancer. The results revealed the following information:

The sample included a relatively young group of women, with caring responsibilities. Five main themes emerged: coping with the rapid transition from 'well' to 'ill', information needs and decision making, needing support and empathy, impact on family, and creating a new 'normal'. More support was needed towards the end of chemotherapy, when side effects were at there most toxic, and decisions about forthcoming surgery were being made. Some women were referred to psychological services, but usually when a crisis point had been reached. ${ }^{11}$

This information is also imperative because it can assist with developing services and creating interventions to meet the complex needs of teaching and educating women about IBC. Most important, it can offer women further understanding of what they may experience as they select to undergo neo-adjuvant chemotherapy for breast cancer, and overall how to survive such experience. 
Historically, IBC treatments consisted of radiation and surgery alone, and yielded a five-year survival rate of $<5 \%$, and in some studies similar to those conducted in the 1950 s, patients had a mean survival rate of approximately 19 months. ${ }^{12}$ Unlike systemic chemotherapeutic agents which attack all cells in their path that are rapidly dividing irrespective of their function, taxanes, are targeted therapies that function in a capacity that disrupts the specific cellular components that are necessary for oncogenesis vis-à-vis carcinogenesis. ${ }^{13}$ This further aids in the combined chemotherapeutic approach to the destruction of cancer cells and helps to improve the low survival rates in the United States, thus diminishing the risk of metastasis. This is especially useful for cases that include patients in which cells have an overproduction of HER2 protein (HER2-positive). ${ }^{1}$

\section{Surgery}

Due to the histology, of IBC, vessels in the breast can become blocked or obstructed by cancerous cells, which accounts for "orange peel" texture and appearance to the skin (peau d'orange), in more advanced cases. ${ }^{1}$ As these cases are more advanced surgery is typically indicated as part of a multimodality approach to cancer treatment, if the tissues respond well to neoadjuvant chemotherapeutic agents. The histological components of IBC affect the breast and surrounding tissues in such a drastic fashion, that breast-conservation surgeries are not an option, therefore making radical mastectomy (including lymph node removal) the best surgical option. ${ }^{1}$ According to Yamauchi et. al., surgery alone historically did not yield what would be deemed cancer free survival rates which are reported as a five-year metric.

\section{Radiation Therapy}

Unlike chemotherapy, radiation therapy is not systemic and only affects the areas of the body in which the radiation beams are aimed. Absent of an intact breast (post-mastectomy), patients will most likely undergo several weeks of radiation therapy treatments to the chest wall and regional lymph nodes in conjunction with additional cycles of chemotherapy. ${ }^{10}$ According to the National Comprehensive Cancer Network (2019) ${ }^{14}$, patients should have a CT-based treatment planning session to identify both the areas of intended treatment and any critical organs that are within proximity to the intended area of treatment.

Patients will undergo approximately 25-28 daily treatments (excluding weekends), or approximately 6 weeks of radiation treatments ${ }^{14}$. The rationale behind the radiation schedule, falls within the law of Bergonie and Tribondeau, which informs clinicians that cells are most susceptible to the effects of radiation while actively dividing. Providing radiation treatments at the same time each day affords the daily doses to catch cells in their most vulnerable state of the cellular reproductive cycle (G2-M phases), and destroy its' DNA. In cases of invasive breast cancers (i.e., more specifically IBC), a combination of different types of radiation may be used to destroy microscopic disease ${ }^{14}$. Another point of view has been advanced by Orecchia, there is better local control of the cancer with the addition of a regimen that includes radiation therapy as radiation therapy alone is reserved for cases in which the breast is unresectable. ${ }^{15}$

\section{Conclusion}

To gain a foothold on breast cancer, the lay community must be empowered with knowledge regarding $I B C$. It is also important to publicize more information regarding IBC within the health care system and breast cancer organizations, plus increase research that may someday develop a screening device for early detection. More aggressive work is needed and funds for breast cancer research are essential and the gateway to making progress against breast cancer. In the meantime, now is the time to utilize health care practitioners, nurses, public health educators, community outreach workers, and additional organizations to continue to promote breast cancer awareness to encourage mammography and SBE (prevention methods), as well as to promote IBC knowledge at the communal level. As a result, education is certainly needed at a community level for all women. In order to abate breast cancer, it is extremely important to implement more aggressive measures that involve educating women throughout the world about IBC and breast cancer. This education will grant a better opportunity to understand the disease, increase early detection, and survival rates of IBC. For further information regarding IBC, you may explore the American Cancer Society, the National Cancer Institute and the Susan G. Komen Breast Cancer Institute. There are more aggressive treatment plans for individuals diagnosed with IBC such as neoadjuvant chemotherapy, targeted and hormone therapy, surgery, and radiation and adjuvant therapy. 


\section{References}

1. American Cancer Society. Cancer facts \& figures 2019. 2019; Atlanta, Georgia. American Cancer Society. Retrieved from http://www.cancer.org/content/dam/cancer-org/research/cancer-facts-andstatistics/annual-cancer-facts-and-figures/2019/cancer-facts-and-figures-2019.pdf

2. Menta A, Fouad TM. Lucci A, et al. Inflammatory breast cancer. Surg Oncol Clin N Am. 2018;98(4): 787-800.

3. National Cancer Institute. Inflammatory Breast Cancer. 2019. Retrieved from: http://www. cancer.gov/ cancertopics/factsheet/Sites-Types/IBC

4. Mei W, Merajver SD. Molecular biology of inflammatory breast cancer: Applications to diagnosis, prognosis, and therapy. BreastDis. 2006; 22(1): 25-34.

5. Morrow RJ, Etemadi N, Yeo B, ErnstM (2017) Challenging a misnomer? The role of inflammatory pathway in inflammatory breast cancer. Hindawi Mediators Inflamm. 2017; Article ID 4754827, https://doi.org/10. 1155/2017/4754827

6. Tungland B.Human microbiota in health and disease: from pathogenesis therapy. 2018; London: England: Academic Press.

7. Taghian A, Merajver SD. Patient education: Locally advanced and inflammatory breast cancer (Beyond and Basics); 2018. Retrieved from: https://www.uptodate.com/contents/locally-advanced-andinflammatory-breast-cancer-beyond-the-basics

8. Susan G. Komen Breast Cancer Institute. Inflammatory breast cancer, 2013. Retrieved from: http://ww5. komen.org/uploadedFiles/Content_Binaries/806-03202a.pdf

9. Susan G. Komen Breast Cancer Institute. Inflammatory breast cancer; 2019. Retrieved from: ww5.komen.org

10. van Uden DJ, van Laarhoven HW, Westenberg AH, de Wilt JH, Blanken-Peeters CF (2015) Inflammatory breast cancer: An overview. Crit Rev Oncol Hemato. 2015;93(2): 116-126.

11. Beaver K, Williamson S, Briggs J. (2016) Exploring patient experiences of neo-adjuvant chemotherapy for breast cancer. Eur J Oncol Nurs. 20: 77-86.doi:10.1016/j.ejon.2015.06.001 [doi]

12. Yamauchi H, Woodward WA, Valero V, et al. (2012) Inflammatory breast cancer: What we know and what we need tolearn. The Oncologist. 2012;17(7): 891-899. doi:10.1634/theoncologist.2012-0039

13. Joo WD, Visintinl, Mora G. Targeted cancer therapy: Are the days of systemic chemotherapy numbered? Maturitas. 2013;76(4): 308-314, doi: 1016/j.maturitas.2013.09.008

14. National Comprehensive Cancer Network. NCCN Clinical practice guidelines in oncology. NCCN Guidelines®. 2019. Retrieved from:https://www.nccn.organd https://www.nccn.org/professionals/ physician_gls/pdf/breast.pdf

15. Orecchia, R. (2018). Radiation therapy for inflammatory breast cancer. European J Oncol Nurs. 2018;44: 1148-1150. 